\title{
Three-Dimensional Computerized Tomography-Assisted Identification of Necrotic Volume, Distribution, Shape and Prognosis of Collapse in ONFH
}

\author{
Jianying Shen ${ }^{1 *}$, Hongyu Wei²*, Qingsheng Yu ${ }^{2,3 \#, ~ L i m i n g ~ C h e n g ² ~}$ \\ ${ }^{1}$ Institute of Chinese Materia Medica, China Academy of Chinese Medical Sciences, Beijing, China \\ ${ }^{2}$ Department of Orthopedics, China-Japan Friendship Hospital, Beijing, China \\ ${ }^{3}$ Alliance PKU Management Consultants Ltd., Beijing, China \\ Email:"yukeisei@163.com
}

Received 27 January 2016; accepted 21 March 2016; published 24 March 2016

Copyright (C) 2016 by authors and Scientific Research Publishing Inc.

This work is licensed under the Creative Commons Attribution International License (CC BY). http://creativecommons.org/licenses/by/4.0/

c) (i) Open Access

\section{Abstract}

Objective: We constructed 3D-model of ONFH in computer according to three-dimensional computerized tomography (3D-CT) data. We determined the location and volume of necrosis to investigate its clinical efficacy. Method: Totally 92 hips (59 cases) with ONFH (44 males, 15 females) were included, with mean age of 37.5 years (range from 26 to 58). Totally 20 cases (35 hips) were induced by corticosteroid (CTSs), 31 (49 hips) induced by alcohol, 4 (4 hips) induced by trauma and 4 (4 hips) idiopathic. All the hips were categorized into stage ARCO II. Finally diagnosed by MRI, all hips were scanned by CT to acquire data in DICOM format. The images were imported into software to extract 3D-shape of femoral heads, necrotic foci, their volumes and distribution in each quadrant. Deviation of volumes between digital image and biopsy specimen was analyzed by SAS9.1 package. Correlativity between collapse and volume of necrosis under specific pathogeneses was also analyzed. Among the cases necessitating total hip arthroplasty (THA) due to advancing to ARCO III, we randomly selected 8 of them to perform 3D-CT scanning thrice prior to surgical operation. Total femoral heads harvested were torn asunder. Cubic capacity of femoral heads and necrotic foci were hereby measured and compared with those acquired from digital models. Result: Through the digital model, necrotic foci were found mainly locating within the super lateral portion of femoral head, coinciding with those observed in biopsy specimen. Average volumetric ratio of digitally acquired necrosis focus/femoral head in 58 collapsed hips was $36.8 \%$. The ratio of the 34 hips without collapse was $17.3 \%$. In collapsed femoral heads, the distribution

\footnotetext{
*J. Shen and H. Wei contribute equally to this article.

${ }^{\sharp}$ Corresponding author.
} 
of necrosis focus was $23.4 \%$ in quadrant 1 (q1), $23.6 \%$ in q2, $12.1 \%$ in q3, $14.4 \%$ in q4, 9.0\% in q5, $11.8 \%$ in $q 6,1.6 \%$ in q7 and $3.9 \%$ in q8. In femoral heads without collapse, the distribution was $34.2 \%$ in $\mathrm{q} 1,29.6 \%$ in $\mathrm{q} 2,11.8 \%$ in $\mathrm{q} 3,11.3 \%$ in $\mathrm{q} 4,6.0 \%$ in $\mathrm{q} 5,6.0 \%$ in $\mathrm{q} 6,0.5 \%$ in $\mathrm{q} 7$ and $0.4 \%$ in q8. As for the average cubic capacities of femoral heads and necrotic foci, those acquired from the digital model and biopsy specimen had no significant difference in matched-pairs test $(t=$ $-1.49, P=0.179$ for femoral heads and $t=-1.52, P=0.172$ for necrotic foci). There was significant difference $(F=2.720, P=0.035<0.05)$ among volumetric ratios of necrotic focus/total femoral head under different pathogeneses, which were $33.55 \%$ in alcohol-induced cases, $26.74 \%$ in the CTSs-induced, $\mathbf{4 0 . 4 1 \%}$ in the trauma-induced, and $18.39 \%$ in idiopathic cases. Shapes of necrotic foci could be subdivided into 5 types: vault, sublobe, dumbbell, ball and petal. The distribution of these 5 types of shape was different among necroses caused by CTSs, alcohol, trauma and idiopathic pathogeneses. The distribution in $\mathbf{5 8}$ collapsed femoral heads was also distinct from that in 34 femoral heads without collapse. Through multinomial logistic regression, we analyzed the correlativity among volumetric ratios of necrotic tissue, its distribution in q2(superioanteriomedial portion of femoral head) and collapse. The result showed statistical significance $(P$ was respectively 0.0001 and 0.0005$)$. Decision tree model showed that $94.6 \%(53 / 56)$ hips would progress into collapse if the volumetric ratio of necrotic tissue was over $23.48 \%$. Otherwise, if distribution in q2 was over $45.13 \%, 83.3 \%(5 / 6)$ hips would progress into collapse. No collapse $(0 / 30)$ would occur if the distribution of necrotic tissue in q2 was under $45.13 \%$. Conclusion: Digital 3D-model reconstructed from CT scanning can precisely incarnate spatial orientation of necrotic foci in femoral head. Multinomial logistic regression and decision-making tree shows that volumetric ratio of necrotic tissues plays an important role in anticipating collapse of femoral head.

\section{Keywords}

Three-Dimensional CT, Collapse of Femoral Head, Osteonecrosis, Digital Three-Dimensional Reconstruction, Decision Tree Analysis

\section{Introduction}

Osteonecrosis of the femoral head (ONFH) is prevalent in orthopedic practice, especially inflicting the young and middle-aged patients. They have similar pathological changes and reparative process, no matter their etiological factors are traumatic or non-traumatic. Without proper treatment, about $80 \%$ femoral head will proceed to collapse within 1 to 4 years after invasion [1] [2]. Once femoral head collapses, it will inevitably develop to ostearthritis. Hip joints will be severely impaired and doom to undergo THA. But its long-term curative effect is not satisfactory because non-traumatic ONFH always inflict young and middle-aged patients with high-demands, which necessitate therapies to reserve the femoral heads. Fortunately, not all the ONFH proceeds to collapse. To prevent or defer collapse through early intervention is feasible to release patients from or postpone THA. Thus the health care cost is saved and suffering from the ill is mitigated. Among the factors leading to collapse, area, cubic capacity and location of necrotic foci are generally accepted as the major ones. After final diagnosis, 3D-CT can further analyze the range of necrotic tissue and assess the collapse, along with detecting the location, size and boundary of necrotic foci. Importing all the data from 3D-CT into computerized imagery and quantitative system, stereoscopic model of femoral head will be reconstructed. Ocular demonstration will be thereby established to identify the location, volume and shape of the necrotic foci in a directly perceived way to facilitate the accuracy and reliability of clinical diagnosis.

\section{Materials and Methods}

\subsection{Materials}

Two measuring graduates with volume of $400 \mathrm{ml}$ and scales divided into $1 \mathrm{ml}$. Two injection syringes with volume of $5 \mathrm{ml}$. Hacksaw with its blade as thin as $1 \mathrm{~mm}$. One straight handheld curette and pointed rongeur forceps. 


\subsection{Selection of Patients}

Fifty-nine cases of ONFH patients in stage of ARCO II (92 hips were inflicted). Forty-four of them are male and fifteen of them are female. Their average age is 37.5 years (from 26 to 58 years old). Twenty cases are induced by CTSs ( 35 hips are involved). Thirty-one cases are induced by alcohol (49 hips are involved). Four cases are induced by trauma (4 hips are involved). Four cases are idiopathic (4 hips are involved).

\subsection{Follow-Up Procedures}

Collapse of femoral heads is the end-point for follow-ups. The non-collapsed femoral heads should be observed for at least 24 months. All the patients are examined by 3D-CT every 6 months. Finally 6 cases (involving 8 hips) are randomly selected from all the collapsed ones. These cases are scanned by 3D-CT before THA. The specimens of femoral heads are taken out during surgical operation, followed by biopsy measurement.

\subsection{Collection of Topographic Data from CT}

Once diagnosed as ONFH in stage of ARCO II by MRI, every patient should have their both hips examined by 3D-CT. Radiographs of hip joints in coronal, axial and sagittal views are hereby acquired. The manufacturer of CT machine is Toshiba (16 layers and multi-lines). Each layer is as thick as $1 \mathrm{~mm}$ with pitch of 1.5. Other parameters include $120 \mathrm{kv}, 260 \mathrm{~mA}$, mAs 187. Window width is $2000 \mathrm{HU}$. Window level is $300 \mathrm{HU}$. Matrix is $512 \times 512$. Hip joints are held in neutral position with flexion to $0^{\circ}$ and external rotation to $10^{\circ}$. Raw data acquired from CT are imported to compact disc in DICOM format.

\subsection{Reconstructing Steric Model of Femoral Head in Computer}

1) Raw data of sliced images in DICOM format are imported into MRIcro software and packed into an img (image) mirror file and an hdr (high-dynamic range) file (Figure 1).

2) The hdr files are opened in our independently developed software as images. The sliced 3D-CT images are imported into the dissection interface in order to identify and discriminate the necrotic tissue from femoral heads.

3) Once priming the dissection, the software automatically enters the dissection interface and generates a lasso tool (Figure 2). Adjust location of the lasso and select 3 point on a fixed plane. Cubic capacity and stereo shape of the femoral head will be automatically generated (Figure 3 and Figure 4).

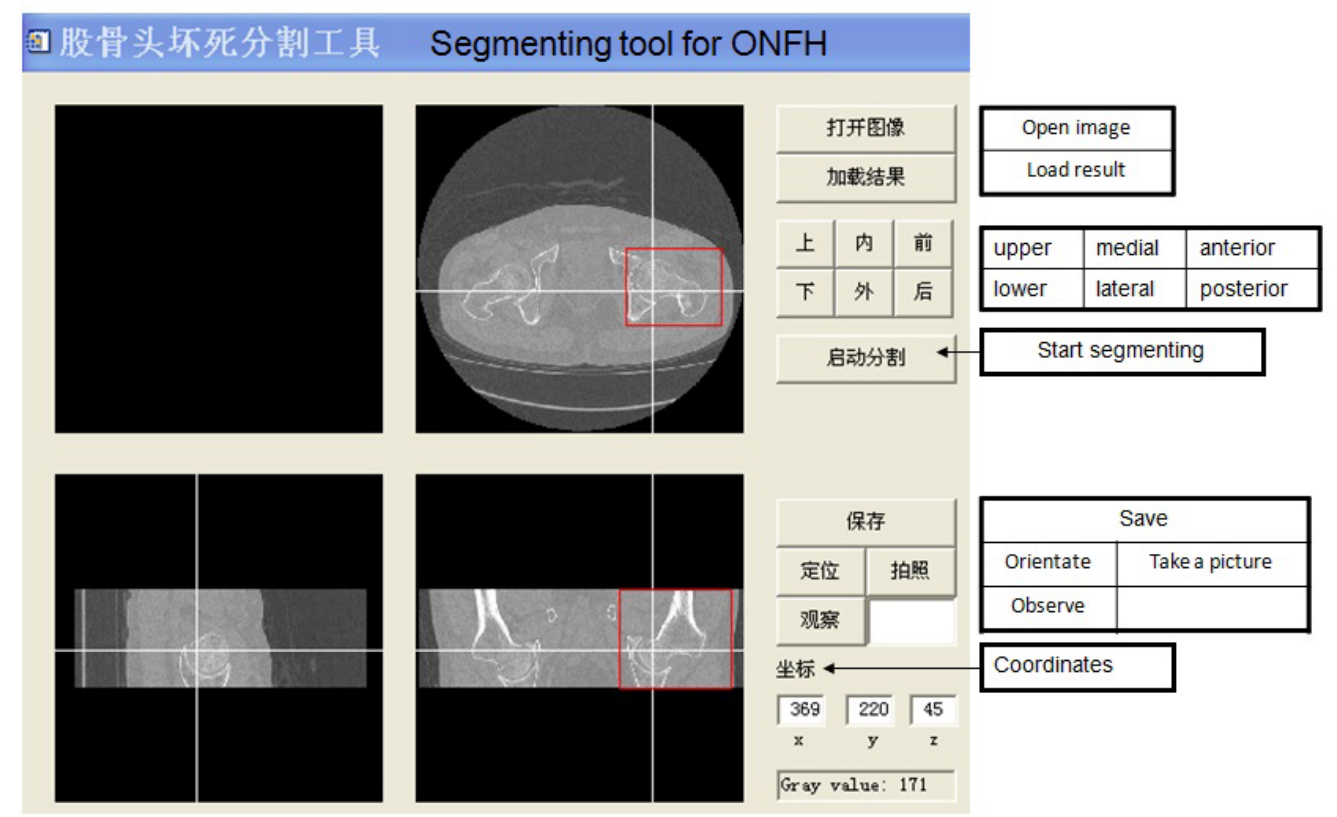

Figure 1. Raw data of CT are imported into computer software. 
分割界面（左侧股骨头） Segmentation interface (left femoral head)

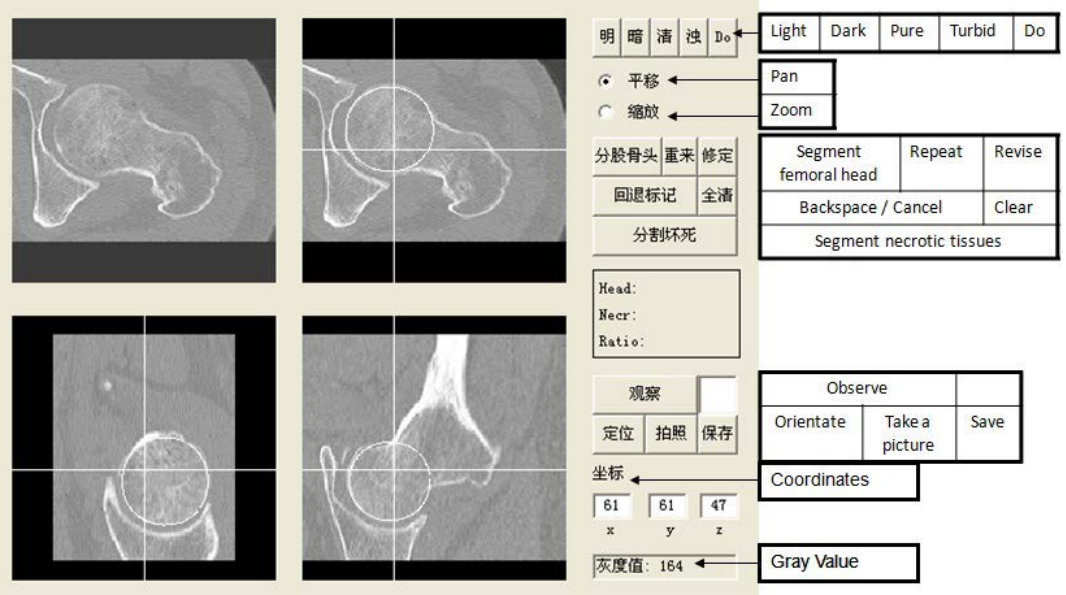

Figure 2. Segmenting femoral head.

\section{分割界面（左侧股骨头） Segmentation interface (left femoral head)}

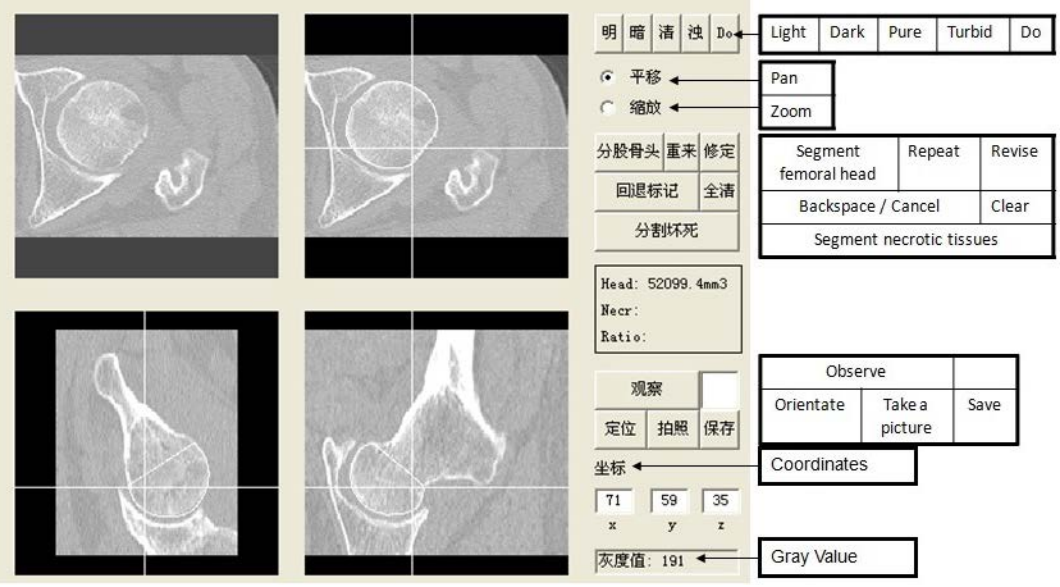

Figure 3. Segmentation and volume of femoral head.

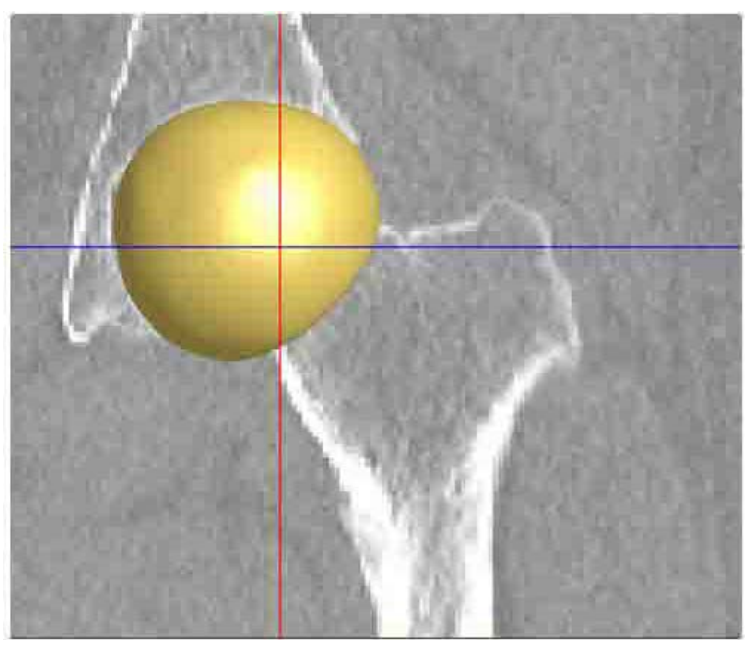

Figure 4. Spatial shape of femoral head. 
4) Identify the borders of necrotic tissues and normal tissues respectively in coronal, sagittal and axial interfaces. Repeat this process thrice in 2 - 3 cutting layers to define normal and necrotic tissues (Figure 5). There is homogeneity within the same tissue and variability on the boundary between different tissues presented by CT. The CT image within one tissue is defined as an assembly of interconnected homogenous pixels with maximal gradient value on its fringe. The computer discerns normal and necrotic tissues via searching for assemblies of pixels in accordance with normal and necrotic tissues. Through subsequent calculation, purchased are cubic capacities for necrotic and normal tissues and the ratio of necrotic tissue to the normal femoral head (Figure 6).

5) Defining quadrants of femoral heads. After segmenting femoral head, software automatically defines its center. The algorithm establishes 3-D coordinates surrounding the center point. Then program orients 8 quadrants in the 3-D coordinates in accordance with the anterior-posterior, upper-lower and medial-lateral directions. Volume of necrotic tissue in each quadrant is calculated, and hence its ratio to the volume of entire necrotic foci. Program marks each quadrant as q1-q8. q1-q4 are anterior. q5-q8 is posterior. Quadrant marked with even number is medial. Those marked with odd number is lateral. q1, 2, 5, 6 are upper quadrants. q3, 4, 7, 8 is lower quadrants.

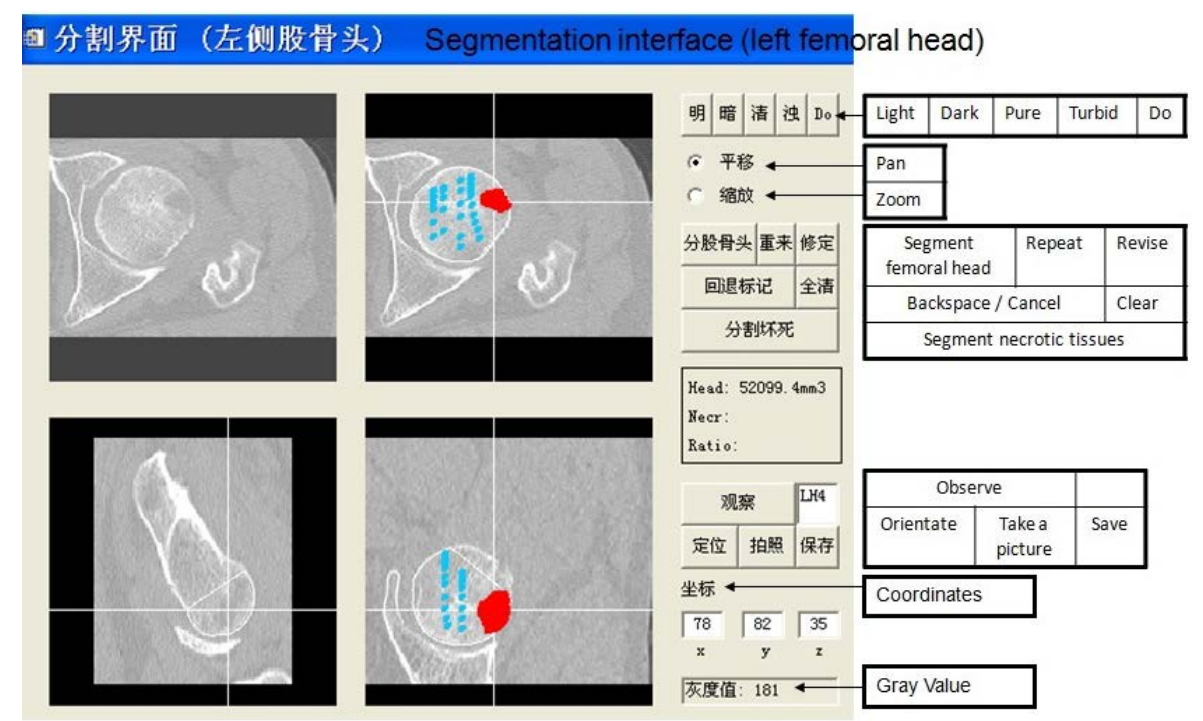

Figure 5. Identifying necrotic focus.

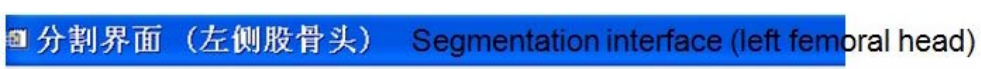

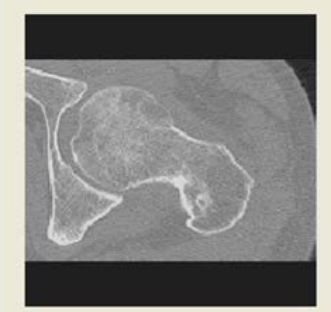
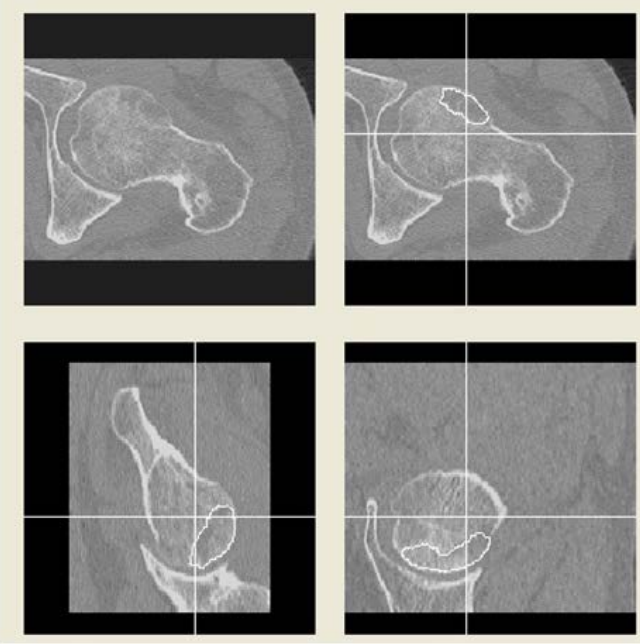

Figure 6. Result of segmenting the necrotic focus.

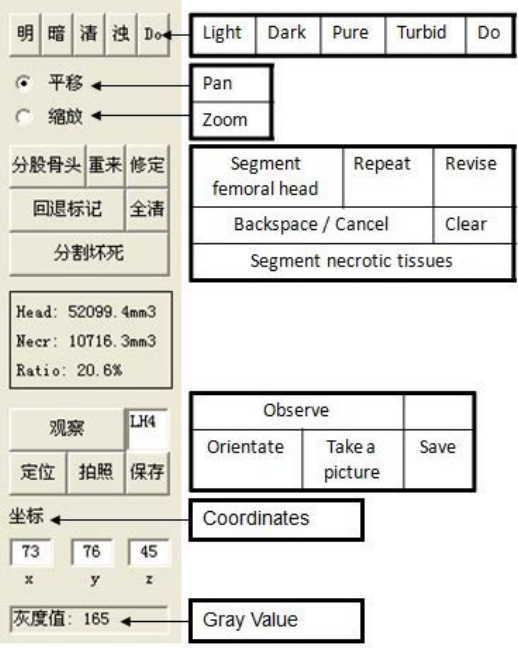

(1) 
6) The software will display distribution of necrotic foci in each quadrant and corresponding proportions in the entire necrotic tissue (Table 1). The software can also display the 3D image of necrotic foci and those respectively in 8 quadrants (Figure 7). The cursor can freely rotate the image to illustrate femoral head, its necrotic foci, their spatial relationship and general distribution in each quadrant. The software can also particularly display the spatial contour of necrotic foci. The cursor can also freely rotate the image to illustrate it in each perspective (Figure 8).

7) All those data can be saved in the main interface of the software. Repeat the abovementioned procedures and save all the manipulated results to assigned folders. Data can be saved in format of raw, sav and txt. The file containing all the information concerning the ONFH can be run either by our software or by other 3D image processing software such as 3Dmax and Amira. The txt file preserves data related with volume of femoral head and necrotic foci, ratio of necrotic foci to femoral head and volumetric proportion of necrotic foci in every quadrant.

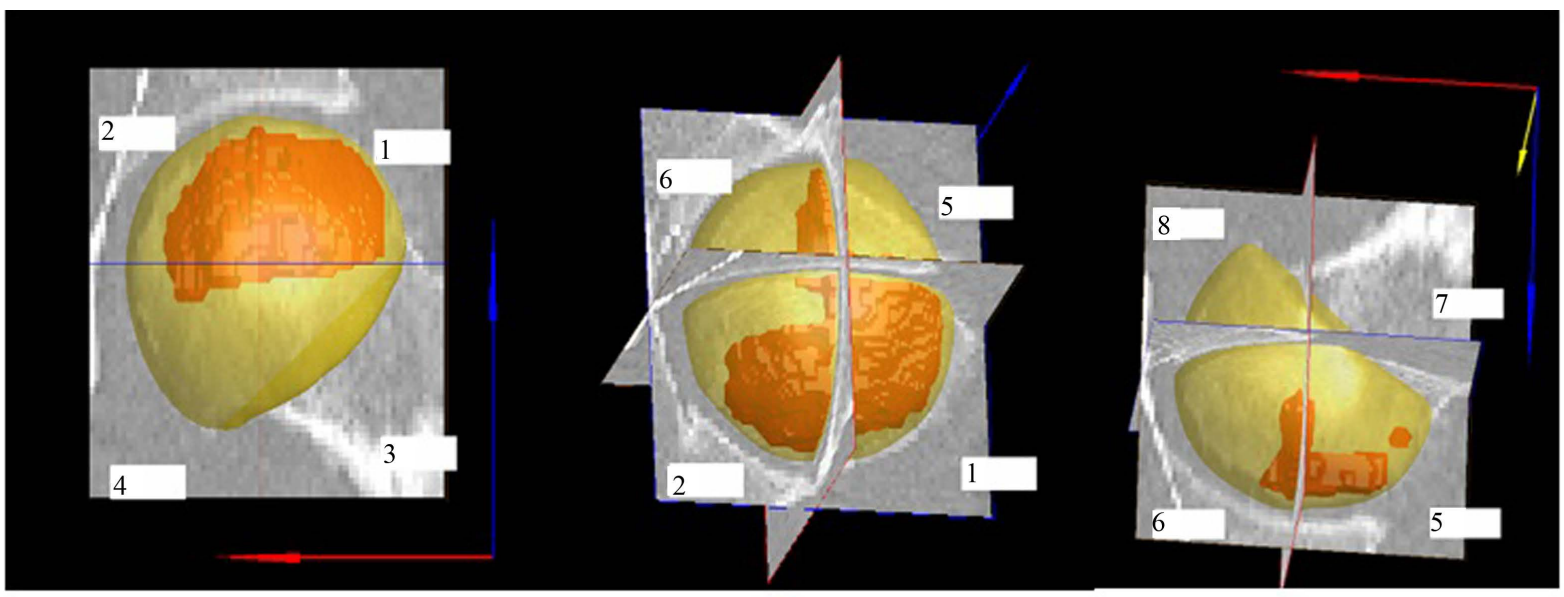

Figure 7. 3D-distribution of necrotic focus in femoral head.

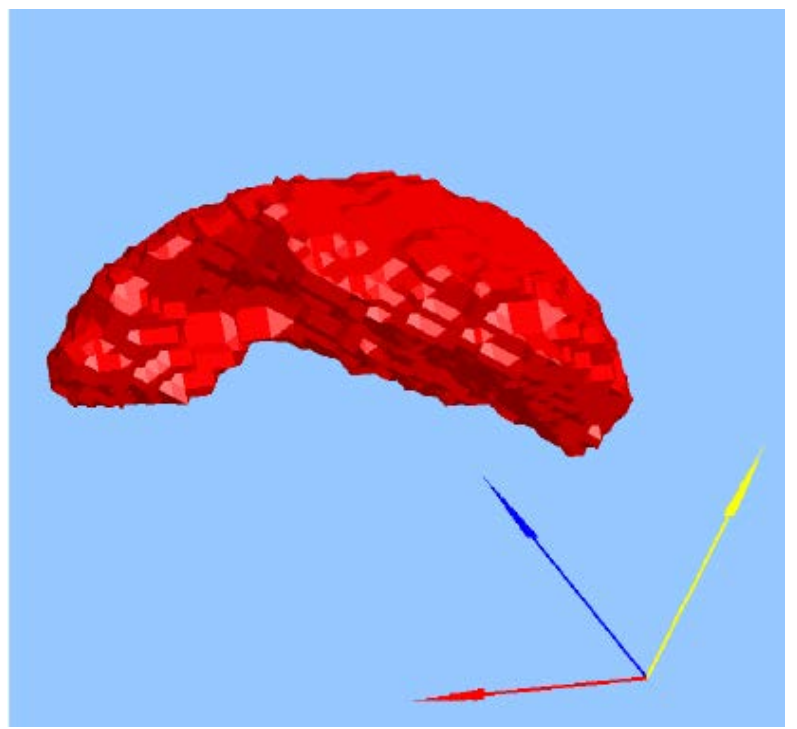

Figure 8. 3D-shape of necrotic focus in femoral head.

Table 1. The proportion (distribution) of necrosis in each quadrant.

\begin{tabular}{ccccccccc}
\hline Quadrant & 1 & 2 & 3 & 4 & 5 & 6 & 7 \\
\hline Proportion (\%) & 50.82 & 17.59 & 24.05 & 0.44 & 2.71 & 2.71 & 0.00 \\
\hline
\end{tabular}




\subsection{Volumetric Measurements for Gross Femoral Heads and Their Necrotic Foci}

From all the patients planned for THA with collapsed femoral heads, six cases (8 hips are involved) are randomly gleaned. Hip joints are scanned with 3D-CT prior to surgical operation for volumetric measuring the femoral head and its necrotic foci by our software. Integral femoral head along with neck is collected during THA operation. Carefully discern and mark the boundary between femoral head and neck. Cut off the femoral head with dynamoelectric saw. Gauge the volume of femoral head and its necrotic foci with measuring glass.

1) Immerse the femoral head into a graduate of normal saline (NS). Adjust the total volume to exactly $300 \mathrm{ml}$. Take out the femoral head with as little NS as possible. Measure the marginal volume of NS remained in the graduate. Repeat the process thrice and reckon the volume of femoral head.

2) Divide the measured femoral head into 5 uniform pieces by dynamoelectric steel saw. Put them into graduate to measure their volumes (Figure 9). Carefully identify the proliferating sclerotic band surrounding necrotic tissue. Use curette and rongeur forceps to eliminate necrotic bone tissue till the sclerotic fringe. Put the remaining pieces into the same graduate. Measure the marginal volume of NS (Figure 10). Volume of femoral head/ necrotic tissue $=300 \mathrm{ml}$-remaining volume. Convert the results to cubic millimeter.
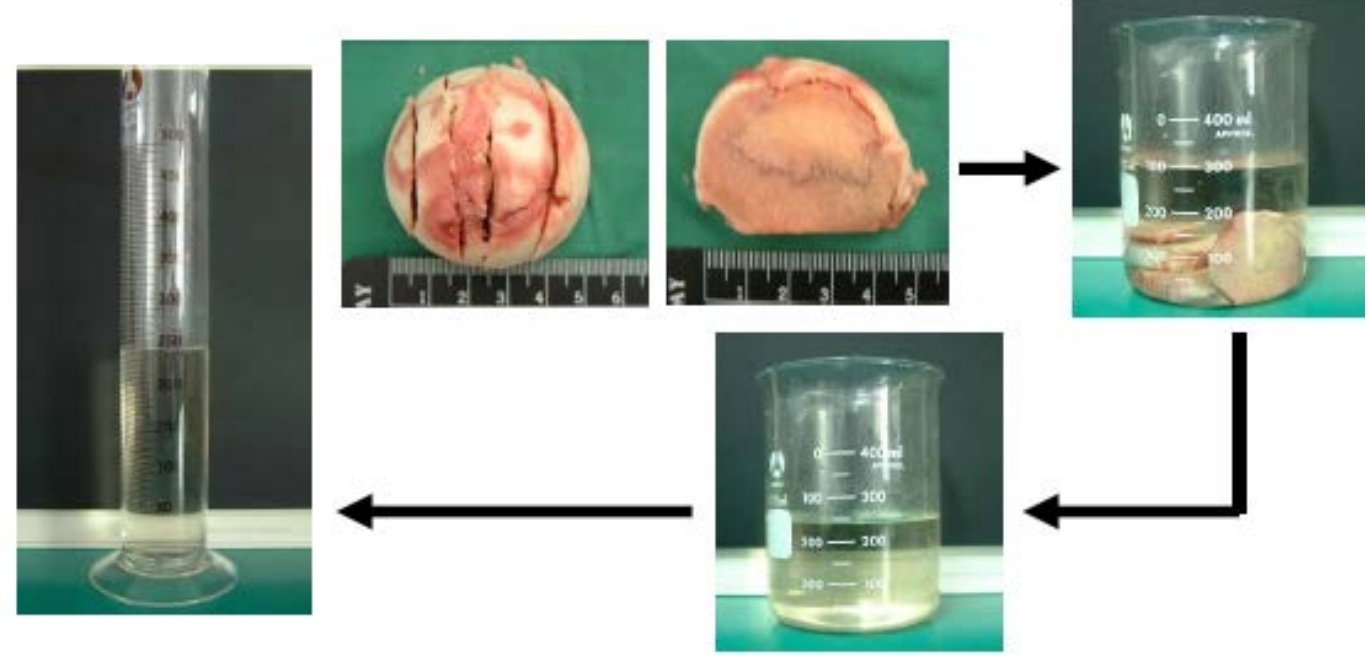

Figure 9. Method for measuring volume of femoral head specimen. Volume of femoral head $\left(\mathrm{cm}^{3}\right)=300$ ml-volume of remaining liquid.
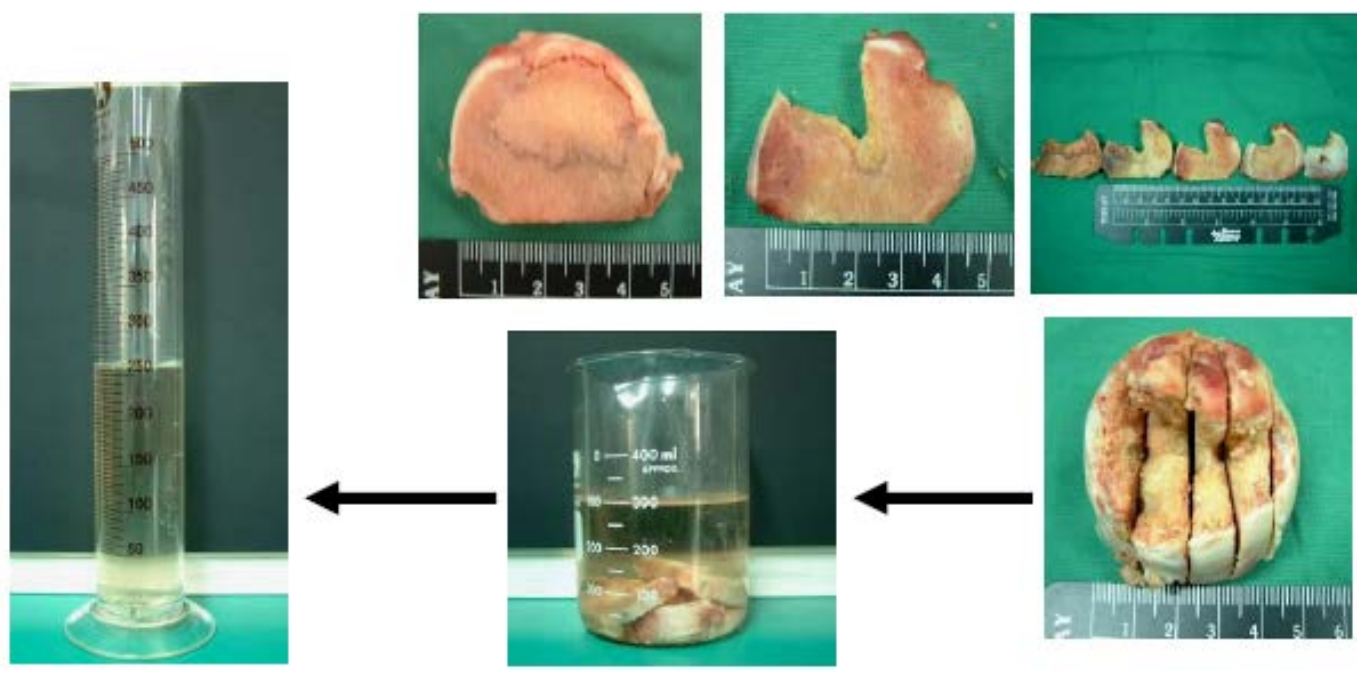

Figure 10. Method for measuring volume of necrotic focus specimen. Volume of necrotic focus $\left(\mathrm{cm}^{3}\right)=300$ ml-volume of remaining liquid. 


\subsection{Statistical Analysis}

Resort all the data got via digital simulation and gross measurement to $t$ test. Use statistical software SAS9.1 to assist the analysis. Use multinomial logistic regression and decision tree analysis to summarize the correlation between every parameters and collapse.

\section{Results}

\subsection{Reconstructing the 3D Model for Femoral Head}

Figure 11 demonstrates a 3D model for femoral head reconstructed by software. The orange part is necrotic region. The yellow region is normal part in femoral head. The necrotic focus locates within the upper-anterior region of the femoral head, coinciding with that observed in gross specimen.

\subsection{Follow-Up Outcomes}

Among the 59 cases (92 hips are involved), totally 37 cases (58 hips) are incurred with collapse of femoral heads. Among them 29 hips are alcohol-induced, 25 are induced by CTSs, 3 by trauma and 1 idiopathic. The rest 22 cases (34 hips are involved) are not suffered from collapse. Among them 20 hips are induced by alcohol, 10 are induced by CTSs, 1 by trauma and 3 idiopathic (Figure 12).

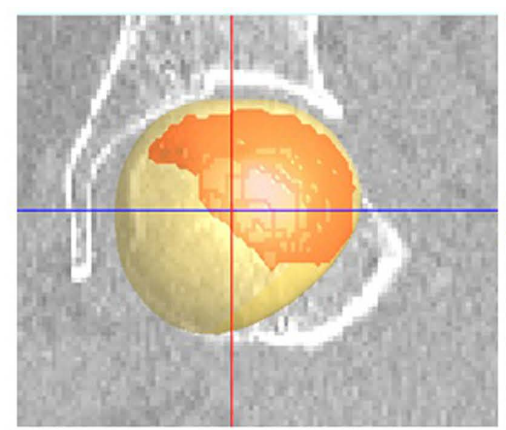

Backward perspective

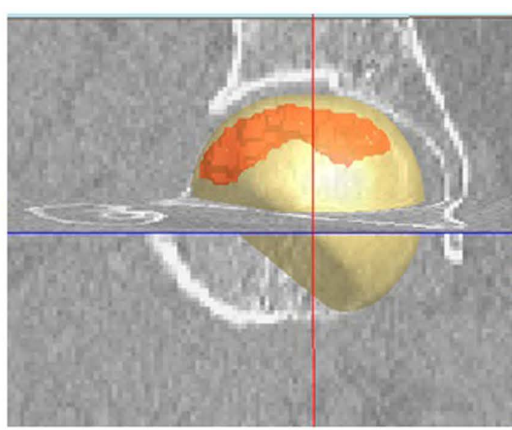

Forward perspective

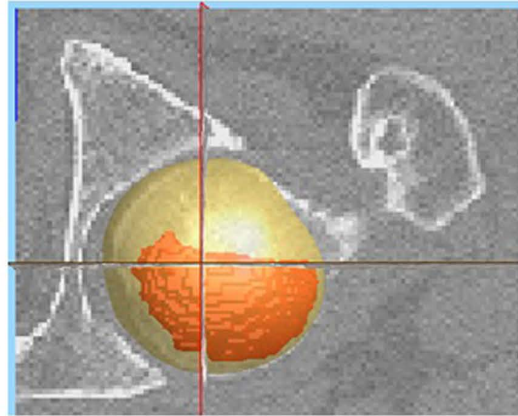

Downward perspective

Figure 11. 3D-model of femoral head.

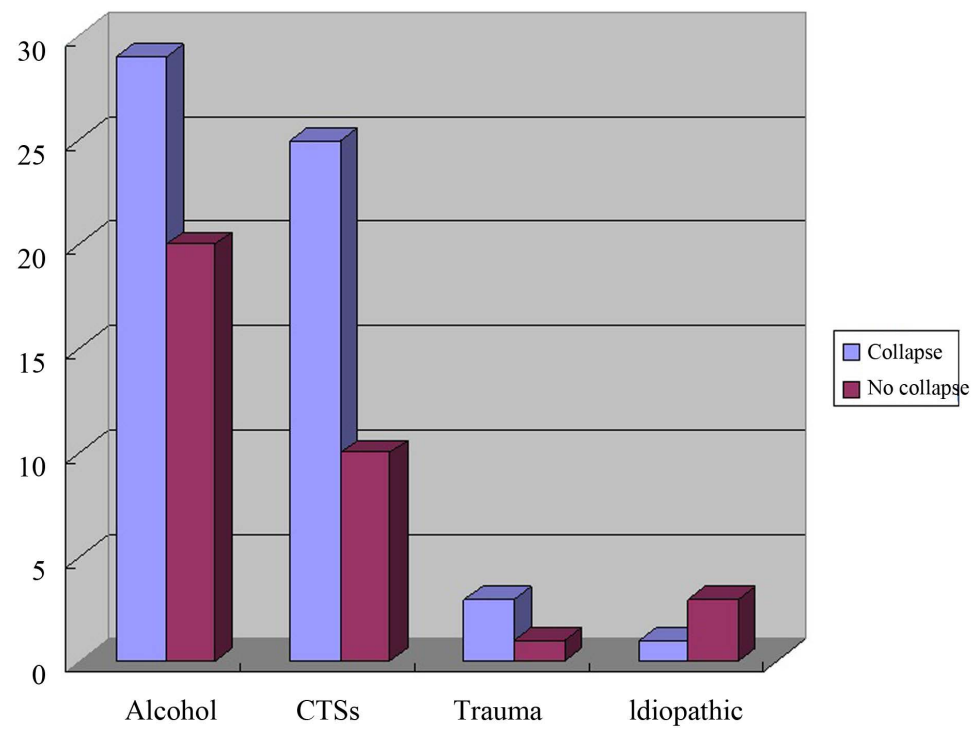

Figure 12. Outcome of ONFH under different pathogenesis. 


\subsection{Comparison between Results Measured in Gross Specimen and Those from Digital Simulation}

Resort the 8 hips planned for THA to 3D-CT scanning before surgical operation. Through mathematical operation, the software outputs volumetric data as $50,251.05 \pm 5630.49 \mathrm{~mm}^{3}$ for femoral heads and 21,756.48 \pm $3065.88 \mathrm{~mm}^{3}$ for necrotic foci. Taken out within surgical operation, the biopsy femoral heads are grossly measured as $50,855.53 \pm 6056.49 \mathrm{~mm}^{3}$ for femoral heads and 22,213.67 $\pm 2785.76 \mathrm{~mm}^{3}$ for necrotic foci. The volumetric value for femoral head got via gross measurement is $604.48 \pm 114.6 \mathrm{~mm}^{3}$ higher than that via software. There is no statistical significance between them (paired $t=-1.49, P=0.179>0.05$ ) (Figure 13). The volumetric value for necrotic foci got via gross measurement is $457.19 \pm 84.9 \mathrm{~mm}^{3}$ higher than that via software. There is no statistical significance between them (paired $t=-1.52, P=0.172>0.05$ ) (Figure 14).

\subsection{Ratio of Necrotic Tissue to Femoral Head (Volume of Necrotic Tissue/Volume of Femoral Head $\times 100 \%$ ) under Different Pathogenesis}

Among the 92 hips inflicted by ONFH, 49 of them are caused by alcohol, with average volumetric ratio of necrotic tissue as $26.74 \% \pm 9.635 \%$ of them are caused by CTSs, with average volumetric ratio of necrotic tissue as $33.55 \% \pm 5.84 \%$ of them are caused by trauma, with volumetric ratio of necrotic tissue as $40.41 \% \pm 10.34 \%$ of them are idiopathic, with volumetric ratio of necrotic tissue as $18.39 \% \pm 4.7 \%$ (Table 2). Result of one factor variance analysis reveals statistical significance among the volumetric ratio of necrotic tissue to femoral head

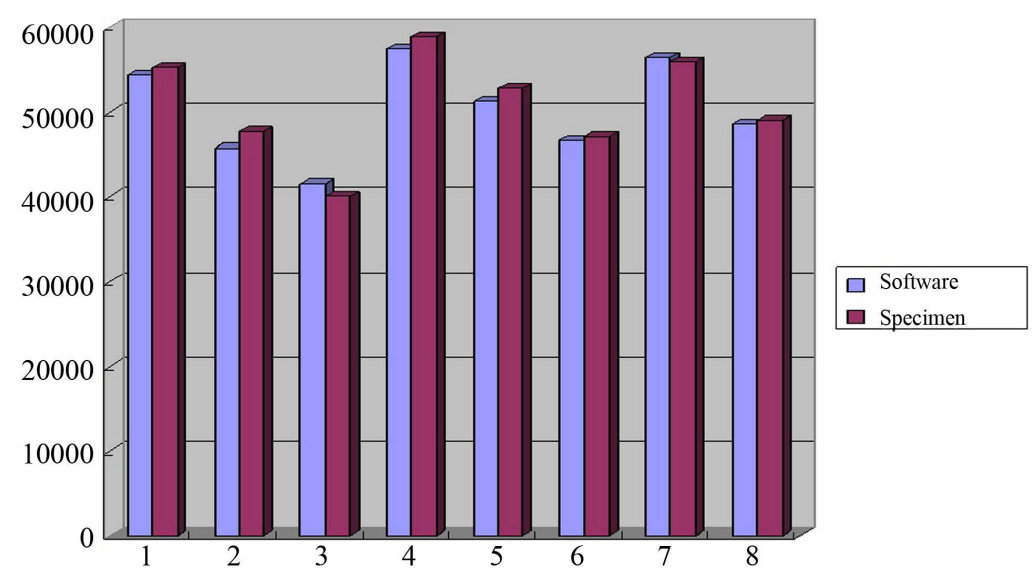

Figure 13. Comparison between volumes of femoral head respectively measured in specimen and software.

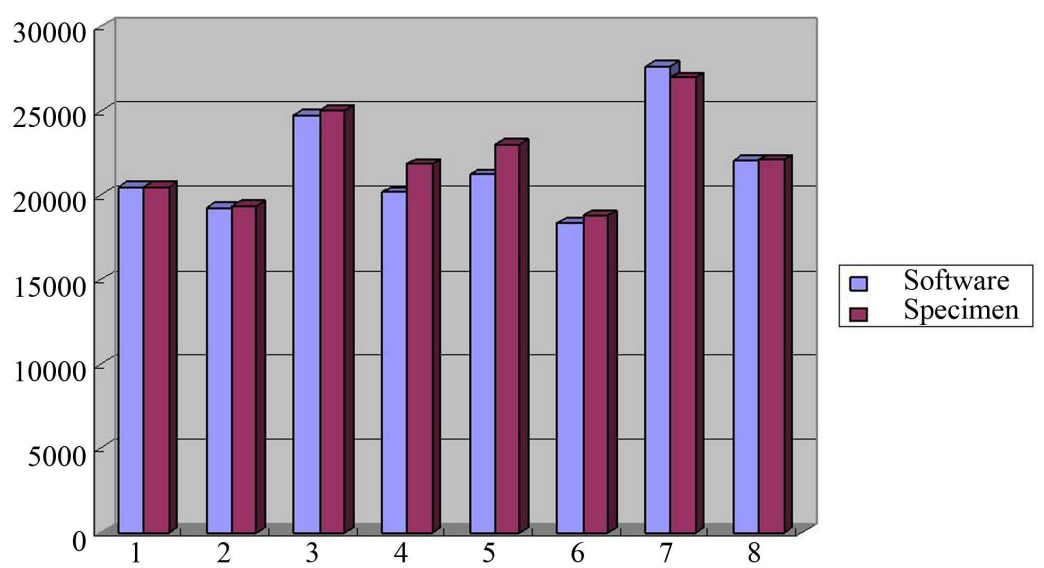

Figure 14. Comparison between volumes of necrotic foci respectively measured in specimen and software. 
Table 2. Volumetric ratios of ONFH under different pathogeneses.

\begin{tabular}{ccc}
\hline Reason & No. of cases & Necrotic vol. ratio (\%) \\
\hline Alcohol & 49 & $33.55 \pm 5.8$ \\
CTSs & 35 & $26.74 \pm 9.6$ \\
Trauma & 4 & $40.41 \pm 10.3$ \\
Idiopathic & 4 & $18.39 \pm 4.7$ \\
Total & 92 & ---- \\
\hline
\end{tabular}

under different pathogenesis $(F=2.720, P=0.035<0.05)$.

\subsection{The Volumetric Ratio of Necrotic Tissues in Femoral Head and the Same Ratio in Each Quadrant}

Average volumetric ratio for the collapsed 58 hips is $36.8 \% \pm 12.5 \%$. That for the not collapsed hips is $17.3 \% \pm$ $5.2 \%$. Among the collapsed femoral heads, necrotic tissue in q1 accounts for $23.4 \% \pm 8.7 \%$ of the entire necrotic tissue. q2 accounts for $23.6 \% \pm 11.7 \%$. q3 accounts for $12.1 \% \pm 8.1 \%$. q4 accounts for $14.4 \% \pm 7.9 \%$. q5 accounts for $9.0 \% \pm 6.8 \%$. q6 accounts for $11.8 \% \pm 7.9 \%$. q7 accounts for $1.6 \% \pm 2.6 \%$. q8 accounts for $3.9 \% \pm$ $5.3 \%$. Among the not collapsed femoral heads, necrotic tissue in q1 accounts for $34.2 \% \pm 5.8 \%$ of the entire necrotic tissue. q2 accounts for $29.6 \% \pm 14.9 \%$. q3 accounts for $11.8 \% \pm 9.8 \%$. q4 accounts for $11.3 \% \pm 12.1 \%$. q5 accounts for $6.0 \% \pm 8.1 \%$. q6 accounts for $6.0 \% \pm 6.8 \%$. q7 accounts for $0.5 \% \pm 2.5 \%$. q8 accounts for $0.4 \% \pm$ $1.3 \%$. Through multinomial logistic regression, volumetric ratios of femoral head are closely related to collapse with $P<0.01$. Ratio of necrotic foci distributed in q2 is also closely related to collapse with $P<0.01$ (Table 3 and Figure 15). We set "end" as dependent variable, with "true" standing for collapse and "false" for not collapse. We set "age", "reason”, "volumetric ratio" and distribution of necrotic foci in q1-q8 as dependent variables. Through decision tree analysis, we find out that the volumetric ratio and the distribution of necrotic foci in q2 are closely related to collapse. There is a watershed when the volumetric ratio $=23.48 \%$. Among the 56 hips with volumetric ratio of necrotic tissue greater than $23.48 \%$, 53 end in collapse. Among the 30 hips with volumetric ratio of necrotic tissue no more than $23.48 \%$ and distribution in q2 no more than $45.13 \%$, no collapse occurs. Among the remaining 6 hips with volumetric ratio no more than $23.48 \%$ and distribution in q2 greater than $45.13 \%, 5$ end in collapse (Figure 16).

\subsection{How Is the Shape of Necrotic Foci Related to Pathogenesis and Collapse?}

The 3D images for necrotic foci of the 92 hips are reconstructed by the measuring software. Observed in different aspects, spatial shape of the necrotic foci can be categorized into vault, sublobe, dumbbell, ball and petal shapes (Figure 17 and Table 4).

Testified by chi-square text, there is no statistical difference among all kinds of shapes of necrotic foci caused by alcohol $\left(X^{2}=5.01, P>0.05\right)$. The same kinds of shapes of necrotic foci in ONFH caused by CTSs, trauma and idiopathic reasons are also cleared of any statistical difference $\left(X^{2}=9.31\right.$ and $P>0.05$ for CTSs, $X^{2}=9.08$ and $P>0.05$ for trauma, $X^{2}=3.46$ and $P>0.05$ for idiopathic reasons). Among the femoral heads end in collapse, there is statistical difference for all the kinds of shapes $\left(X^{2}=9.51,0.01<P<0.05\right)$. There is no statistical difference among the femoral heads without collapse $\left(X^{2}=4.35, P>0.05\right)$.

\section{Discussion}

ONFH usually progresses latently, characterized by ischemia of medulla and necrosis of bone trabeculae. CT and MRI are important measures to diagnose ONFH in early stage. MRI is particularly sensitive to ONFH in early stage and discovers it ahead of other means. Although the deviation is too large to accurately incarnate the volume of necrotic foci, there are some measures reported to forecast collapse of femoral head via iconographic analysis. Some other means have high precision but their practicality is compromised. Insufficient quantitative methods to orientate and measures volume of necrotic foci leads to controversy about treatment for ONFH. Our research intends to explore an efficacious, intuitive and practical way to accurately measure the volume, dis- 
J. Y. Shen et al.

Table 3. The logistic procedure summary of stepwise selection.

\begin{tabular}{cccccccc}
\hline & Effect & \multicolumn{2}{c}{ Number } & \multicolumn{2}{c}{ Score } & \multicolumn{2}{c}{ Variable } \\
\hline Step & Entered & DF & In & Chi-Square & Pr $>$ ChiSq & Label \\
1 & volume & 1 & 1 & 41.8192 & $<0.0001$ & volume \\
2 & q 2 & 1 & 2 & 10.1273 & 0.0015 & $\mathrm{q} 2$ \\
\hline
\end{tabular}

Table 4. The distribution of necroses in femoral head.

\begin{tabular}{|c|c|c|c|c|c|c|}
\hline & Alcohol & CTSs & Trauma & Idiopathic & Collapsed & Non-collapsed \\
\hline Vault & 21 & 23 & 1 & 1 & 30 & 16 \\
\hline Sublobe & 6 & 3 & 0 & 0 & 4 & 5 \\
\hline Dumbbell & 7 & 2 & 0 & 0 & 6 & 3 \\
\hline Ball & 8 & 6 & 3 & 2 & 12 & 7 \\
\hline Petal & 7 & 1 & 0 & 1 & 6 & 3 \\
\hline Total & 49 & 35 & 4 & 4 & 58 & 34 \\
\hline
\end{tabular}

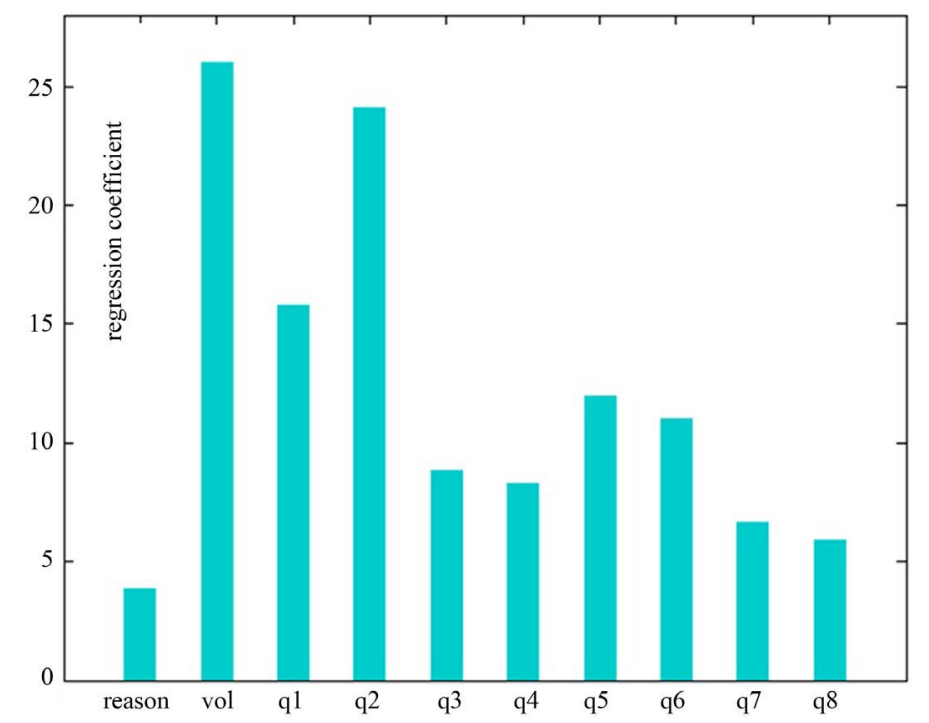

Figure 15. Regression coefficient of every parameter in logistic regressive equation.

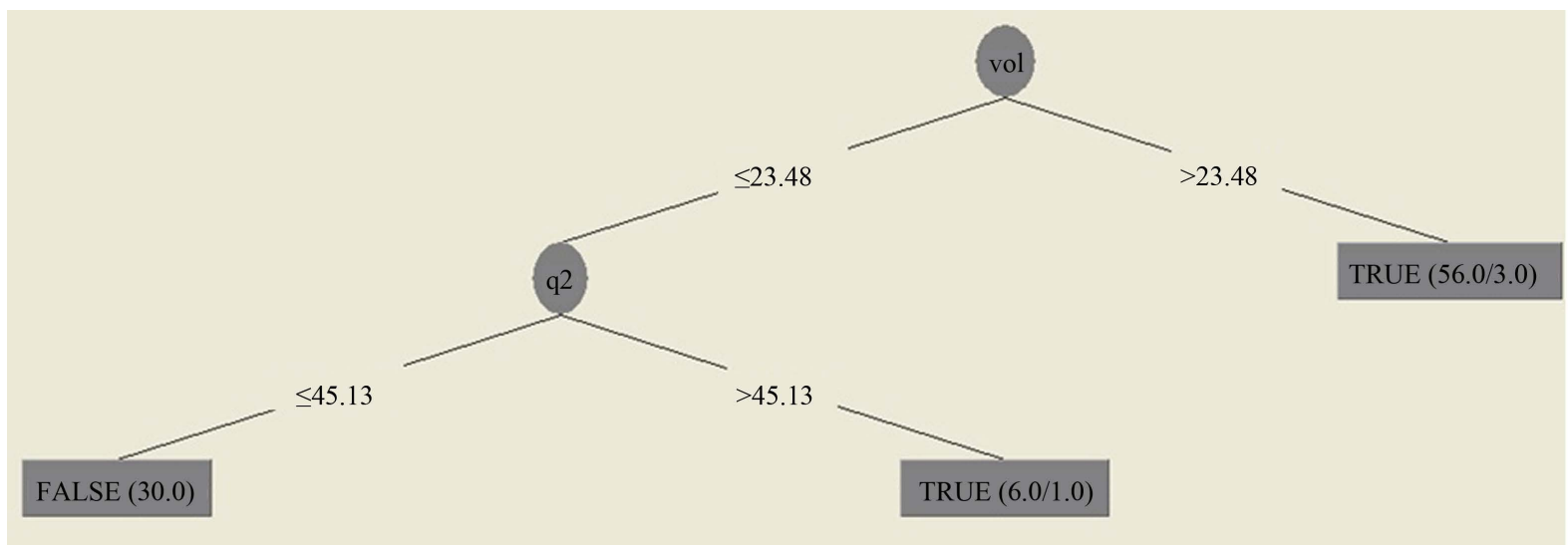

Figure 16. Analytic result from decision tree. 


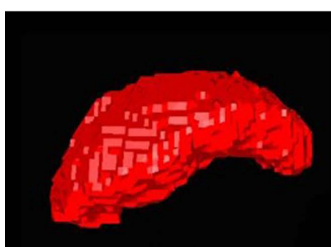

(a)

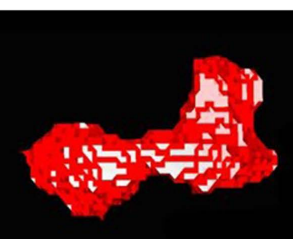

(b)

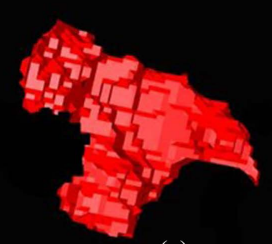

(c)

(d)

(e)

Figure 17. Shapes of ONFH. (a) Vault, (b) Sublobe, (c) Dumbbell, (d) Ball, (e) Petal.

tribution and shape of necrotic foci and therefore to forecast the risk of collapse.

\subsection{Forecasting Risk of Collapse by Measuring the Area of Necrotic Tissue}

1) Kerboul et al. reported the method of summing angles of necrotic tissues in 1974 [3]. They measured the angles of the affected area in anteroposterior film and lateral radiograph. Scale of affected area is indicated by sum of the two angles. They concluded that if sum of the two angles is greater than $200^{\circ}$, collapse is very likely to happen. Otherwise, if it is less than $160^{\circ}$, risk of collapse decreases to bring about better prognosis.

2) Steinberg et al put forward staging method in 1984 to reflect the volume of necrotic tissues [4]. The ARCO staging system which is widely used nowadays also reflected the volume of necrotic tissues. It uses Roman numerals I, II, III, IV to indicate the stage and degree of necrosis. Letters A, B and C indicate area of necrosis. A indicates necrotic area less than $15 \%$. B is between $15 \%$ and $30 \%$. C is greater than $30 \%$.

3) Sugano et al. put forward the superficial ratio method in 1994 [5] [6]. They performed a retrospective study on a group of patients based on the percentage of necrotic foci in entire femoral head surveyed by anteroposterior film and lateral radiograph. After 3 years of follow-up, they made two conclusions. One is that the percentage of necrotic tissues in collapse group is significantly greater than that in the group without collapse. The other is that the critical value for collapse is contingent on the position of necrosis.

These abovementioned methods are easy to popularize because they are based on mere plain films. But they cannot actually reflect the volume and position of necrotic foci. Moreover, prognosis based on mere plain film cannot duly and precisely precaution collapse because plain film cannot reflect necrotic characters until it reaches stage II, more often stage III [7].

4) Based on coronal T1-weighted MR image, risk of collapse can be judged on the signal and proportion of necrotic tissue to the weight-bearing surface. Shimizu et al studied 66 hips suffered from ONFH. After averagely 32 months of follow-up, 32\% resulted in collapse [2] [8]. All these collapsed femoral heads had their necrotic tissues extending beyond one third of the weight-bearing surface. Among the femoral heads with their necrotic tissues extending beyond two thirds of the weight-bearing surface and one fourth diameter of femoral head, 74\% resulted in collapse. They also found out that collapsing risk is the highest if T1-weight image presents mixed signal. The risk decreases with high signal. The lowest risk is associated with low signal.

5) Measuring percentage of necrotic area, necrotic index and modified necrotic index based on MRI.

Measure to segment out tissues of interest has been reported by Li et al. A CT scan was done on mandible. This CT dataset in DICOM format was imported into Mimics 10.0 software in which a 3-D model of the facial skeleton was reconstructed and the mandible was segmented out [9]. Hereby percentage of necrotic area is measured by dividing the area of necrotic tissue by the entire femoral head in coronal and sagittal T1-weighted MRI. Through retrospective study on 56 cases of which the femoral heads were preserved, Li et al. found out that T1-weighted MRI can be used to forecast collapse of femoral heads in stage I and II of ONFH [10]. There is 
high risk when the necrotic percentage is greater than $60 \%$. The risk is medium when it is between $30 \%$ and $60 \%$. The femoral head is somewhat free of collapse if the percentage is below $30 \%$. Index for the extent of necrosis $=(\mathrm{A} / 180) \times(\mathrm{B} / 180) \times 100$. Center point of femoral head is set as reference on the intermediate layer of T1-weighted MRI, angles for the necrotic regions are measured as A on coronal plane and B on sagittal plane. The layer involving maximal region of necrosis substitutes the intermediate layer as for the modified index for the extent of necrosis [11]. Comparing the abovementioned 3 methods, Cherian et al. thought the modified index for the extent of necrosis is the most reliable. However, difficulties to judge the layer involving maximal region of necrosis and to measure the extent thereof weakens it operability [12].

\subsection{Forecasting Collapse through Measuring Volume of Necrotic Regions in Femoral Head}

It is difficult to accurately describe and quantify the location and volume of necrotic foci in femoral head no matter through plain film or MRI. Consequently, prognosis preservation of femoral head cannot be authentically estimated. Hernigou et al. delineated boundaries between necrotic and normal tissues on MRI and input it into software. Volume of necrotic foci is got by sum of multiplying area of necrotic foci by depth of stratum [13]. Because boundary between necrotic and normal tissue is characterized by serpiginous low signal zones on T1-weight MRI, the authors believe that MRI can accurately measure the volume of necrotic foci with low error rate.

Thanks to development of computer technique, Mahdian et al. performed 3D analysis of the temporomandibular joint (TMJ) of patient undergoing surgery with subtotal TMJ replacement. They gave a suitable formulation of mathematical model, which describes the changes of stresses in TMJ incurred after the surgery [14]. Earlier, Nishii et al. reconstructed 3D image of necrotic foci in 2002 [15]. They adopted measures similar to that of Hernigou except for using 3D coordinate to indicate extent and distribution of necrotic foci. After 2 years of follow-ups with 65 hips in 47 cases and multiple regression analysis, they concluded that volume of necrotic tissues significantly related to collapse. There is high risk of collapse if the ratio of necrotic tissue is greater than $30 \%$. Risk of collapse closely relates to center of gravity of the necrotic foci if the ratio is less than $30 \%$. Collapse is imminent if the center of gravity of necrotic focus situates between $40^{\circ}$ and $70^{\circ}$ of latitude and $50^{\circ}$ and $100^{\circ}$ of longitude, which is the anterior upper position of femoral head.

After comparing abovementioned ways forecasting collapse of femoral head, David R. Steinberg believes that it is the most reliable to measure the volume of necrotic tissue [16]. Measuring the angle of necrotic tissue has the greatest error. While the method invented by Hernigou or Nishii is too difficult to manipulate for clinical practice, which is deemed by the inventors themselves [15].

The software developed in our research is oriented by clinical practice and guided by iconographic characters of ONFH. Based on grey level of necrotic and normal tissues, we constructed 3D model of femoral head and calculated the volumetric ratio of necrotic foci to entire femoral head. Besides, distribution of necrotic foci in each quadrant and their shape facilitate forecasting collapse of femoral head. By visualizing the spatial shape and location of necrotic foci, this software can help surgeons precisely cleaning up necrosis. Measuring each sample thrice can minimize errors, which accords with reports by Hernigou and Nishii [15].

In current research, we forecast collapse of femoral head according to volume of necrotic foci. Through multinomial logistic regression and decision tree analysis, collapse of femoral head is found to closely relate to volumetric ratio and distribution in quadrant 2 of necrotic tissue and it is statistically significant. In order to remove any human factor affecting the result, the data were processed with decision tree analysis. The result coincides with Nishii's. Therefore, detecting volume, shape and location of necrotic foci with 3D graphics is valuable to forecast collapse of femoral head with necrotic foci of small or medial scale.

\subsection{Volume and Etiology of Femoral Head}

Computer can setup and modify models to calculate and simulate musculoskeletal bodies and forces thereof and to transfer them to a finite element model for etiological and prognostic analyses. Kunze et al. created pelvis model from a hemipelvis CT dataset to examine different effects when standing up from three different seat heights [17]. As for our study, despite numerous etiologies believed to underlie ONFH, none of them is definite. There is no report regarding consistency of etiology with extent and volume of necrosis. Studying 58 hips of ONFH, Zhao et al. found that necrosis often occurred in fossa of femoral head and juncture between femoral head and neck and shared similar pathological characters without statistical difference as for extent, thickness 
and hyperplasia [18]. Through angiographic study, Atsumi et al. found that all ischemic necrosis in ONFH was caused by angiemphraxis of feeding artery [19].

In our study, we found statistical difference for volumetric ratio of necrosis among ONFH caused by different pathogenesis. Some researchers believe that volume of necrosis in traumatic ONFH hinges on level and extent of feeding artery damaged [20]. Volume of necrosis in traumatic ONFH is obviously bigger than those caused by other pathogenesis because the superior retinacular ramus of medial circumflex femoral arteries is very likely to be damaged by trauma. Therefore, it will incur large scale necrosis because this impaired blood supply cannot be compensated by other arteries [21]. Nonetheless, the limited traumatic cases available in our study could cause statistical error, which necessitates further study to reveal correlation between necrotic volume and pathogenesis.

\subsection{Shape of Necrosis and Risk of Collapse}

The shape of ischemic necrosis of femoral head has seldom been reported. We believe that the shape is related with distribution and scope of damaged vessels. No matter caused by trauma or not, impaired blood supply is major pathogenesis for ischemic necrosis in ONFH [22]. Sevitt et al. found out that blood supply in femoral head consisted of minute blood vessel formed by rami of major vessels such as superior and inferior retinacular artery [23]. Each vessel has its supplying realm relative independent from others although there are vascular anastomoses. In the anatomical perspective, blood supply in femoral head is relatively poor. Once vessels supplying femoral head are damaged by pathological factors such as CTSs, collateral circulation cannot be effectively established. Mean while, because of the special vascularity of femoral head, retinacular arteries form basilar artery ring at the junction between head and neck of femur [24]. They form arched arterial rete via vascular anastomosis at the deep stratum of articular cartilage, where they form anastomoses with nutrient and epiphyseal arteries [25].

Because of the special vascularity in femoral head, ischemic necrosis tends to happen when the impaired vascularity cannot be compensated. As for the vault-shaped necrosis which subchondrally distributes, it could be ischemic necrosis caused by damage of subcartilaginous vault-shaped arterial rete originated from retinacular artery. As for the ball-shaped necrosis, we found that the necrotic range was extended, distribution of which coincided with multilayer arched arterial rete. We think it is caused by damage of corresponding arteries. Among the 4 cases of traumatic necrosis in our study, 3 present this shape because superior retinacular artery is usually damaged in traumatic ONFH [26]. Once this artery is damaged, blood supplied by the arched arterial rete comprising its terminal branches is interrupted. The wide-bound ischemia incurred thereby will lead to bulky necrotic foci equiform with femoral head. The dumbbell-shaped and sublobe-shaped necrotic foci could be caused by arterial injury in different distribution area. With further damage of blood supply, the necrotic foci connected. Sometimes it also could be result of compensation by other arteries. The petal-shaped necrosis appears smooth in cross-section CT. There is obvious continuous sclerotic zone along its borderline, suggesting its correlation with focal reparation.

Regarding collapse, there is statistical difference among different shapes of necrosis. Vault-shaped necrosis is the most apt to collapse, followed by ball-shaped necrosis. Their curved surfaces are parallel with and close to the cartilaginous surface of femoral head and have bulkier foci. Therefore they bear stronger compressive stress.

\subsection{Principle for Distinguish and Segment Necrosis in CT Imagery by Software}

Thanks to successful application of diagnostic image in clinical practice, image segmentation plays a more and more important role in it. Kale et al. reported automatic image methods to segment human facial tissue which contains very thin anatomic structures, applying to images from both MRI and CT [27]. Such method is not only indispensable to extract quantitative information of special tissues from images but also premise and preprocessing of visualization. Because of its principle and specificity of its objectives, medical imaging is affected by noise, field deflection and local action. It is also inevitably marked by blur and inhomogeneity. Moreover, complicated anatomical structure and individual difference conflict difficulties upon medical image segmentation. Conventional imaging techniques are doomed to fail or be specially processed. Therefore some researchers put forward some practical algorithms for segmentation in recent years [28].

CT image is made up of pixels, each of which has a CT value to reflect X-ray absorptivity and attenuation of tissues. There are some algorithms independent of pixel such as Lloréns et al. presents a new automatic method 
based on fuzzy connectedness object extraction and mathematical morphology processing to estimate the path of the nerve and cross-sectional views to segment the jaw tissues [29]. While our method is based on different CT value of pixels tissues because of their distinct absorptivity and attenuation to X-ray. The CT value of pixels in the same tissue is approximately homogenous. Pixels contrast each other across the border of different tissues, which endows CT to discriminate different tissues. Gradient value reflects the disparity among pixels. Greater the gradient value is, more possible it is the pixel locates on the edge of different tissues. Object on CT image is defined as assembly of connected pixels, with homogenous values inside it and maximal gradient value on the edge of it. We identify objects on CT image complying with this definition. Segmenting different objects on medical image should follow their individual characteristics. There is no such a method universally suitable for segmenting all objects. We adopt the method of deformable surface to segment femoral head [30]. This method can search for assembly of pixels with the greatest gradient value by adjusting its form. When the summed gradient value is maximal on the surface, it is the boundary between necrotic and normal tissues. Method of deformable surface is the major topic in medical image segmentation. It is proved optimal to identify object with distinct borders in medical image, therefore suitable to segment femoral head. Segmenting necrotic region is implemented by the method of region growing and graph cut. The principle for region growing is to construct region dot by assembling pixels with similar property. Those surrounding the seed pixels with similar property are merged into one region. In our study, red seed pixel grows in necrotic region and blue seed pixel grows in normal region. This method is featured with efficiency, accuracy and simple computation. Principle for the method of graph cut is to segment image via graph theory, which regards every pixel as a node of the image. Graph cut is to seek assembly of connected nodes with the most homogenous CT value interiorly and sharp contrast on the border. Graph cut arises in recent years on account of its good effect on the realm of computer vision and medical image segmentation [31]. Graph cut is suited for segmenting necrotic region in femoral head because it can promptly and accurately segment objects with irregular shape. To maximize its practicality, we gave priority to automation, foolproof and economization when developing this medical image segmenting software. Given 130 pieces of picture in DICOM format in a 3D-CT of femoral head, our software can accomplish discrimination and segmentation within 3 minutes.

\subsection{Applying Decision Tree Analysis to Medical Statistics}

Present medical diagnosis remains within the confines of actual inspection, which is to identify specific pathology. Nowadays clinicians need to investigate interactions between indices when system biology gradually substitutes for reductionism. Data mining provides new technology for this issue.

Decision tree algorithm is a subfield of data mining, suitable for categorizing high dimensional data [32]. It is qualified with promptness, precision and simply generative scheme. It is inductive learning algorithm based on clinical instance, inferring classification rules in form of decision tree from a set of disordered and random instance. It adopts the pattern of recursive descent, comparing attribute values at internal node, judging descending branch from this node according to respective attribute values and arriving at a conclusion at leaf node. Therefore the path from root to leaf node corresponds with a conjunctive law and the entire decision tree corresponds with a group of laws for disjunctive expressions.

Compared with other categorizing methods, decision tree is swift, accurate and easily transformed to classifying rules. Decision tree approaches in common use include J48, ID3, C4.5 and CART. Among them, J48 is all-important one and widely used in medical data. Chen et al. established diagnostic tree via combining symptoms and diagnostic message with accuracy over 90\% [33]. Its advantages include rapidly establishing intuitive and terminal diagnostic system from medical data. Meanwhile it provides accuracy to facilitate clinical diagnosis and attribution of terminal events. In our research, we utilized J48 decision tree to perform data mining, thus screening out indices most correlated with collapse of femoral head, such as volumetric ratio of necrotic tissues in femoral head and its distribution in quadrant 2 . The major-minor relation among these indices should be harmonized. The volumetric ratio of necrotic tissues to entire femoral head should be in major order where it is greater than $23.48 \%$ and distribution of necrotic tissues in quadrant 2 should be in minor order. Here is the point of inflection for their major-minor correlation. When the volumetric ratio of necrotic tissue is less than $23.48 \%$, the deciding factor switches to distribution of necrotic tissues in quadrant 2 . Hence q2 holds the major order until it is less than $45.13 \%$. When distribution of necrotic tissue in q2 is less than $45.13 \%$, there is no collapse and vice versa. A simple model is thus created to present comprehensibly different prognoses, which few data analy- 
sis method could parallel.

Computer-aided image analyses have been implemented to disentangle many orthopedic etiologies such as instability mechanisms for dislocation under reproducible, physiologically realistically boundary condition. Herrmann et al. introduced a novel testing approach by means of a hardware-in-the-loop (HiL) simulation involving a highly flexible mechatronic testing system to enable comparable investigations of different total hip and knee replacements [34]. Besides, computerized 3D reconstruction of CT image can be utilized to measure and locate necrotic foci in femoral head. This method can distinguish lesion in early stage through abnormal structure of trabeculae such as cluster and stellate formation. Besides, its competitive edge lies in revealing correlation between necrotic foci and surrounding structures. Although not as sensitive as MRI to early pathology, 3D-CT can judge progression of disease and less time-consuming and costly. Equipped with image segmentation and automatic recognition, it needs less manual operation than those reported by other literature, availing to its popularization [30].

There is also some fault in our study. We used ONFH in stage ARCO II, which limited the availability for cases. We used collapse in stage III diagnosed by image as endpoint. Most patients had undergone decompression operation on medulla heretofore, which could exert an influence upon the final result. Some error could occur when software automatically identifies configuration on CT image. To classify shapes of necrotic tissues requires a large number of samples which is not sufficient enough in our research.

\section{Conclusions}

1) CT imaging software can precisely reconstruct 3D-model of femoral head, assisting orthopedic clinician intuitively and spatially apprehend distribution, location and shape of necrotic tissues in femoral head.

2) Based on the 3D-model of ONFH, we can precisely figure out the volume of necrotic tissue and its distribution in each quadrant. This result is reliable and tallies with that measured on specimen.

3) Our study shows that collapse of femoral head closely relates to volume of necrotic tissues. Risk of collapse is as high as $94 \%$ when volumetric ratio of necrotic tissue exceeds $23 \%$.

4) Location of necrotic tissue plays a pivotal role in progression of disease when volume of necrotic tissue is less than $23 \%$. Risk of collapse of is as high as $83 \%$ if $45 \%$ of necrotic tissue locates within anterior-medial-upper section (quadrant 2).

5) The mechanism for different pathogeneses incarnating themselves in distinguish shapes is inexplicit. It may be correlated with lesion of blood vessels in different location. Overall necrotic foci in vault shape are susceptible to collapse.

6) Furnished with modern techniques such as medical image segmentation and automatic recognition, measuring software can nimbly divide and calculate the volumes of femoral head and its necrotic foci. Its application is instructive in forecasting prognoses for ONFH which confounds orthopedic practitioners.

\section{Acknowledgements}

The authors appreciate the assistance from Dr. Zi-Rong Li, MD, the honorary director of Center for Osteonecrosis and Joint Preserving and Reconstruction, Department of Orthopedics, China-Japan Friendship Hospital, Beijing, China.

\section{References}

[1] Ohzono, K., Saito, M., Takaoka, K., Ono, K., Saito, S., Nishina, T., et al. (1991) Natural History of Nontraumatic Avascular Necrosis of the Femoral Head. The Journal of Bone and Joint Surgery British, 73, 68-72.

[2] Sakamoto, M., Shimizu, K., Iida, S., Akita, T., Moriya, H. and Nawata, Y. (1997) Osteonecrosis of the Femoral Head: A Prospective Study with MRI. The Journal of Bone and Joint Surgery British, 79, 213-219. http://dx.doi.org/10.1302/0301-620X.79B2.7179

[3] Kerboul, M., Thomine, J., Postel, M. and Merle d'Aubigne, R. (1974) The Conservative Surgical Treatment of Idiopathic Aseptic Necrosis of the Femoral Head. The Journal of Bone and Joint Surgery British, 56, 291-296.

[4] Steinberg, M.E., Brighton, C.T., Steinberg, D.R., Tooze, S.E. and Hayken, G.D. (1984) Treatment of Avascular Necrosis of the Femoral Head by a Combination of Bone Grafting, Decompression, and Electrical Stimulation. Clinical Orthopaedics and Related Research, 186, 137-153. http://dx.doi.org/10.1097/00003086-198406000-00023

[5] Sugano, N., Takaoka, K., Ohzono, K., Matsui, M., Masuhara, K. and Ono, K. (1994) Prognostication of Nontraumatic 
Avascular Necrosis of the Femoral Head. Significance of Location and Size of the Necrotic Lesion. Clinical Orthopaedics and Related Research, 155-164.

[6] Aaron, R.K., Lennox, D., Bunce, G.E. and Ebert, T. (1989) The Conservative Treatment of Osteonecrosis of the Femoral Head. A Comparison of Core Decompression and Pulsing Electromagnetic Fields. Clinical Orthopaedics and Related Research, 209-218.

[7] Nishii, T., Sugano, N., Ohzono, K., Sakai, T., Haraguchi, K. and Yoshikawa, H. (2002) Progression and Cessation of Collapse in Osteonecrosis of the Femoral Head. Clinical Orthopaedics and Related Research, 149-157. http://dx.doi.org/10.1097/00003086-200207000-00019

[8] Shimizu, K., Moriya, H., Akita, T., Sakamoto, M. and Suguro, T. (1994) Prediction of Collapse with Magnetic Resonance Imaging of Avascular Necrosis of the Femoral Head. The Journal of Bone and Joint Surgery American, 76, 215-223.

[9] Li, P., Tang, Y., Li, J., Shen, L., Tian, W. and Tang, W. (2013) Establishment of Sequential Software Processing for a Biomechanical Model of Mandibular Reconstruction with Custom-Made Plate. Computer Methods and Programs in Biomedicine, 111, 642-649. http://dx.doi.org/10.1016/j.cmpb.2013.05.024

[10] Sun, W., Li, Z.R., Shi, Z.C., Zhang, N.F. and Zhang, Y.C. (2006) Changes in Coagulation and Fibrinolysis of PostSARS Osteonecrosis in a Chinese Population. International Orthopaedics, 30, 143-146. http://dx.doi.org/10.1007/s00264-005-0067-6

[11] Koo, K.H. and Kim, R. (1995) Quantifying the Extent of Osteonecrosis of the Femoral Head. A New Method Using MRI. The Journal of Bone and Joint Surgery British Volume, 77, 875-880.

[12] Cherian, S.F., Laorr, A., Saleh, K.J., Kuskowski, M.A., Bailey, R.F. and Cheng, E.Y. (2003) Quantifying the Extent of Femoral Head Involvement in Osteonecrosis. The Journal of Bone and Joint Surgery American Volume, 85-A, 309-315. http://www.ncbi.nlm.nih.gov/pubmed/12571310

[13] Hernigou, P. and Lambotte, J.C. (2001) Volumetric Analysis of Osteonecrosis of the Femur. Anatomical Correlation Using MRI. The Journal of Bone and Joint Surgery British Volume, 83B, 672-675. http://dx.doi.org/10.1302/0301-620X.83B5.11447

[14] Mahdian, N., Dostalova, T., Danek, J., Nedoma, J., Kohout, J., Hubacek, M., et al. (2013) 3D Reconstruction of TMJ after Resection of the Cyst and the Stress-Strain Analyses. Computer Methods and Programs in Biomedicine, 110, 279-289. http://dx.doi.org/10.1016/j.cmpb.2012.12.001

[15] Nishii, T., Sugano, N., Ohzono, K., Sakai, T., Sato, Y. and Yoshikawa, H. (2002) Significance of Lesion Size and Location in the Prediction of Collapse of Osteonecrosis of the Femoral Head: A New Three-Dimensional Quantification Using Magnetic Resonance Imaging. Journal of Orthopaedic Research, 20, 130-136. http://dx.doi.org/10.1016/S0736-0266(01)00063-8

[16] Steinberg, D.R., Steinberg, M.E., Garino, J.P., Dalinka, M. and Udupa, J.K. (2006) Determining Lesion Size in Osteonecrosis of the Femoral Head. The Journal of Bone and Joint Surgery American Volume, 88, 27-34. http://dx.doi.org/10.2106/JBJS.F.00896

[17] Kunze, M., Schaller, A., Steinke, H., Scholz, R. and Voigt, C. (2012) Combined Multi-Body and Finite Element Investigation of the Effect of the Seat Height on Acetabular Implant Stability during the Activity of Getting Up. Computer Methods and Programs in Biomedicine, 105, 175-182. http://dx.doi.org/10.1016/j.cmpb.2011.09.008

[18] Zhao, F.C., Li, Z.R., Zhang, N.F., Wang, B.L., Sun, W., Cheng, L.M., et al. (2010) Lesion Size Changes in Osteonecrosis of the Femoral Head: A Long-Term Prospective Study Using MRI. International Orthopaedics, 34, 799-804. http://dx.doi.org/10.1007/s00264-009-0829-7

[19] Atsumi, T. and Kuroki, Y. (1992) Role of Impairment of Blood Supply of the Femoral Head in the Pathogenesis of Idiopathic Osteonecrosis. Clinical Orthopaedics and Related Research, 277, 22-30.

[20] Steffen, R.T., Athanasou, N.A., Gill, H.S. and Murray, D.W. (2010) Avascular Necrosis Associated with Fracture of the Femoral Neck after Hip Resurfacing: Histological Assessment of Femoral Bone from Retrieval Specimens. The Journal of Bone and Joint Surgery British Volume, 92B, 787-793. http://dx.doi.org/10.1302/0301-620X.92B6.23377

[21] Konishiike, T., Makihata, E., Tago, H., Sato, T. and Inoue, H. (1999) Acute Fracture of the Neck of the Femur: An Assessment of Perfusion of the Head by Dynamic MRI. The Journal of Bone and Joint Surgery British Volume, 81B, 596-599.

[22] Drescher, W., Schneider, T., Becker, C., Hobolth, L., Ruther, W., Bunger, C., et al. (1999) Reperfusion Pattern of the Immature Femoral Head after Critical Ischemia: A Microsphere Study in Pigs. Acta orthopaedica Scandinavica, 70, 439-445. http://dx.doi.org/10.3109/17453679909000978

[23] Sevitt, S. and Thompson, R.G. (1965) The Distribution and Anastomoses of Arteries Supplying the Head and Neck of the Femur. The Journal of Bone and Joint Surgery British Volume, 47, 560-573.

[24] Schoenecker, P.L., Lesker, P.A. and Ogata, K. (1984) A Dynamic Canine Model of Experimental Hip Dysplasia. Gross 
and Histological Pathology, and the Effect of Position of Immobilization on Capital Femoral Epiphyseal Blood Flow. The Journal of Bone and Joint Surgery American Volume, 66, 1281-1288.

[25] Zlotorowicz, M., Szczodry, M., Czubak, J. and Ciszek, B. (2011) Anatomy of the Medial Femoral Circumflex Artery with Respect to the Vascularity of the Femoral Head. The Journal of Bone and Joint Surgery British Volume, 93B, 1471-1474. http://dx.doi.org/10.1302/0301-620X.93B11.26993

[26] Trueta, J. and Harrison, M.H.M. (1953) The Normal Vascular Anatomy of the Femoral Head in Adult Man. The Journal of Bone and Joint Surgery British Volume, 35B, 442-461. http://www.ncbi.nlm.nih.gov/pubmed/13084696

[27] Kale, E.H., Mumcuoglu, E.U. and Hamcan, S. (2012) Automatic Segmentation of Human Facial Tissue by MRI-CT Fusion: A Feasibility Study. Computer Methods and Programs in Biomedicine, 108, 1106-1120. http://dx.doi.org/10.1016/j.cmpb.2012.07.006

[28] Bezdek, J.C., Hall, L.O. and Clarke, L.P. (1993) Review of MR Image Segmentation Techniques Using Pattern Recognition. Medical Physics, 20, 1033. http://dx.doi.org/10.1118/1.597000

[29] Llorens, R., Naranjo, V., Lopez, F. and Alcaniz, M. (2012) Jaw Tissues Segmentation in Dental 3D CT Images Using Fuzzy-Connectedness and Morphological Processing. Computer Methods and Programs in Biomedicine, 108, 832-843. http://dx.doi.org/10.1016/j.cmpb.2012.05.014

[30] Zoroofi, R.A., Nishii, T., Sato, Y., Sugano, N., Yoshikawa, H. and Tamura, S. (2001) Segmentation of Avascular Necrosis of the Femoral Head Using 3-D MR Images. Computerized Medical Imaging and Graphics, 25, 511-521. http://dx.doi.org/10.1016/S0895-6111(01)00013-1

[31] Top, A., Hamarneh, G. and Abugharbieh, R. (2011) Active Learning for Interactive 3D Image Segmentation. In: Fichtinger, G., Martel, A. and Peters, T., Eds., Medical Image Computing and Computer-Assisted Intervention, MICCAI 2011, 14th International Conference, Toronto, 18-22 September 2011, 603-610. http://dx.doi.org/10.1007/978-3-642-23626-6_74

[32] Alnahi, H. and Alshawi, S. (1993) Knowledge Discovery in Biomedical Databases: A Machine Induction Approach. Computer Methods and Programs in Biomedicine, 39, 343-349. http://dx.doi.org/10.1016/0169-2607(93)90037-L

[33] Fazakerley, J., Charnock, P., Wilde, R., Jones, R. and Ward, M. (2010) Application of a Statistical Software Package for Analysis of Large Patient Dose Data Sets Obtained from RIS. Radiation Protection Dosimetry, 139, 455-458. http://dx.doi.org/10.1093/rpd/ncq105

[34] Herrmann, S., Kaehler, M., Souffrant, R., Rachholz, R., Zierath, J., Kluess, D., et al. (2012) HiL Simulation in Biomechanics: A New Approach for Testing Total Joint Replacements. Computer Methods and Programs in Biomedicine, 105, 109-119. http://dx.doi.org/10.1016/j.cmpb.2011.07.012 